\title{
Nucleation of vortex pairs in exchange biased nanoelements
}

A. S. M. Silva, Ana L. Dantas, G. O. G. Rebouças, and A. S. Carriço

Citation: Journal of Applied Physics 109, $07 D 314$ (2011);

View online: https://doi.org/10.1063/1.3537924

View Table of Contents: http://aip.scitation.org/toc/jap/109/7

Published by the American Institute of Physics

\section{Articles you may be interested in}

Controlling the core-to-core distance of vortex pairs in exchange-biased iron elliptical nanoelements Journal of Applied Physics 111, $07 D 102$ (2012); 10.1063/1.3670978

Tailoring the vortex core in confined magnetic nanostructures

Journal of Applied Physics 111, 07D116 (2012); 10.1063/1.3675989

Giant magnetocaloric effect of thin Ho films

Journal of Applied Physics 109, 07A914 (2011); 10.1063/1.3549566

Controlling the vortex core of thin Permalloy nano-cylinders dipolar coupled to Co polarizers

Journal of Applied Physics 115, $17 D 110$ (2014); 10.1063/1.4860056

Dipolar field effects on the critical current for spin transfer switch of iron and permalloy nanoelements Journal of Applied Physics 115, 17D130 (2014); 10.1063/1.4865317

\section{Scilight} Sharp, quick summaries illuminating the latest physics research 


\title{
Nucleation of vortex pairs in exchange biased nanoelements
}

\author{
A. S. M. Silva, ${ }^{1, a)}$ Ana L. Dantas, ${ }^{1, b)}$ G. O. G. Rebouças, ${ }^{2}$ and A. S. Carriço ${ }^{3}$ \\ ${ }^{1}$ Department of Physics, UERN, Mossoró, RN, Brazil 59.610-210 \\ ${ }^{2}$ Department of Physics, UFERSA, Angicos, RN, Brazil 59515-000 \\ ${ }^{3}$ Department of Physics, UFRN, Natal, RN, Brazil 59072-970
}

(Presented 17 November 2010; received 24 September 2010; accepted 21 October 2010; published online 24 March 2011)

\begin{abstract}
We report a theoretical investigation of interface effects in the magnetic order of interface biased iron and Permalloy ${ }^{\mathrm{TM}}$ elliptical nano-elements. Contrary to intuition, there is a partial pinning of the interface layer, favoring double vortex states along the hysteresis loop. Interface biasing affects the relative chirality and the distance of the vortices. Unbiased nanoelements may nucleate vortex pairs with the same chirality separated by an antivortex. For interface biased nanoelements the vortex pair forms with opposite chirality separated by a magnetic domain. (C) 2011 American Institute of Physics. [doi:10.1063/1.3537924]
\end{abstract}

There are two interesting ways of tailoring the magnetic states and hysteresis loops of soft ferromagnetic materials. Patterning ferromagnetic materials in small nano-elements leads to an enhancement of demagnetizing field effects, favoring the formation of flux closure states, notably the vortex state of circular nano-elements and the Landau state of square nanoelements. ${ }^{1,2}$ On the other hand, by exchange coupling a ferromagnetic material to a large uniaxial anisotropy antiferromagnetic substrate, one adds an extra source of exchange energy, favoring the formation of uniform states. ${ }^{3,4}$

Experimental results for Permalloy ${ }^{\mathrm{TM}}$ biased elliptical dots show that the nature of the magnetization reversal is mostly determined by the shape anisotropy and the exchange bias plays a lesser role compared to the circular dots. However, for certain combinations of aspect ratios and applied fields, varying the direction of the exchange bias can trigger a change of the magnetization reversal from a reversal via vortex formation to a reversal dominated by magnetization rotation. ${ }^{5}$

Interface biased patterned nanostructured systems offer a particularly interesting scenario for the investigation of geometrical constrictions, as well as exchange bias, on the magnetic phases and reversal mechanisms of ferromagnetic materials. Recent work on exchange biased patterned structures has, most often, focused on systems with constrictions of the order of micrometers. There are reports claiming that exchange bias inhibits vortex formation and others indicating the presence of vortexlike features in the hysteresis loops. ${ }^{6}$

In this paper we report a theoretical investigation of the nucleation of vortex pairs along the hysteresis loops of interface biased iron $(\mathrm{Fe})$ and Permalloy ${ }^{\mathrm{TM}}$ (Py) elliptical nanoelements with an aspect ratio around 0.5 , and height from 20-30 nm and lateral dimensions of a few hundred nanometers. For iron ${ }^{7}$ and Permalloy ${ }^{\mathrm{TM}} 8$ the exchange lengths are $11.2 \mathrm{~nm}$ and $15.5 \mathrm{~nm}$. The chosen geometrical dimensions are of the order of a several exchange lengths. Thus,

\footnotetext{
a) Also at Department of Physics, UFRN, Natal, RN, Brazil 59072-970.

${ }^{b)}$ Author to whom correspondence should be addressed. Electronic mail: dantasal@gmail.com. Fax: +558432078789.
}

there is room for formation of nonuniform states within the layers parallel to the interface.

The point of choosing Fe and Py elliptical nano-elements with dimensions in the near-100 nm range is that under these geometrical confinement conditions the nucleation multiple vortices states or domain walls is unlikely. Furthermore, flux closure states are most likely formed in nano-elements with large saturation magnetization. Therefore it might be interesting to investigate exchange biased soft nano-elements with large and small saturation magnetization.

Interface bias is aimed at producing stabilization of a uniform magnetic structure in the nano-element. It is therefore intuitive to expect that the stronger the interface field strength, the more uniform the magnetic state of the nano-element, and the more likely it is to have square hysteresis curves.

We show presently that, contrary to intuition, the interface field induces the nucleation of vortex pairs along the hysteresis curve. Furthermore, interface bias affects the relative chirality and the distance between the vortices.

The uniaxial anisotropy easy axis is parallel to the interface field along the in-plane $x$ axis, and we assume that the antiferromagnetic substrate is held frozen in the antiferromagnetic order. The magnetic structure is described in terms of the magnetic moments of small cubic cells with edge $d$ smaller than the exchange length. The energy density is given by:

$$
\begin{aligned}
E= & \frac{A}{d^{2}} \sum_{j} \sum_{k}\left(1-\hat{m}_{j} \cdot \hat{m}_{k}\right)-M_{S} \vec{H}_{\mathrm{int}} \cdot \sum_{i} \hat{m}_{i} \\
& -M_{S} \vec{H} \cdot \sum_{j} \hat{m}_{j}-K \sum_{j}\left(m_{j}^{x}\right)^{2} \\
& +\frac{M_{S}^{2}}{2} \sum_{j} \sum_{k}\left(\frac{\hat{m}_{j} \cdot \hat{m}_{k}}{n_{j k}^{3}}-\frac{3\left(\hat{m}_{j} \cdot \hat{n}_{j k}\right)\left(\hat{m}_{k} \cdot \hat{n}_{j k}\right)}{n_{j k}^{5}}\right)
\end{aligned}
$$

where the first term is the intrinsic exchange energy, restricted to nearest-neighbor cells and $A$ is the ferromagnetic exchange stiffness. The second term is the interface exchange energy, and the sum is restricted to the first layer of cells at the interface. The third term is the Zeeman energy, the fourth term is the anisotropy energy, and the last term is the 
TABLE I. Magnetic parameters.

\begin{tabular}{llc}
\hline \hline & $\mathrm{Fe}^{\mathrm{a}, \mathrm{b}}$ & $\mathrm{Py}^{\mathrm{c}}$ \\
\hline$M s(A / \mathrm{m})$ & $1.7 \times 10^{6}$ & $0.8 \times 10^{6}$ \\
$A(\mathrm{~J} / \mathrm{m})$ & $2.5 \times 10^{-11}$ & $1.3 \times 10^{-11}$ \\
$K\left(\mathrm{~J} / \mathrm{m}^{3}\right)$ & $4.7 \times 10^{-4}$ & zero \\
\hline \hline
\end{tabular}

${ }^{\mathrm{a}}$ Reference 11.

${ }^{\mathrm{b}}$ Reference 12 .

${ }^{\mathrm{c}}$ Reference 13 .

magnetostatic energy. $M_{S}$ is the saturation magnetization, $\hat{m}_{i}$ is the direction of the magnetic moment of the $i$ th cell, and $n_{i j}$ is the distance between the cells $i$ and $j$ in units of cell size $d$. We use a simulation cell with edge $d=3 \mathrm{~nm}$, for iron, $d=5$ $\mathrm{nm}$ for Permalloy ${ }^{\mathrm{TM}}$, and the values for the iron and Permalloy ${ }^{\mathrm{TM}}$ parameters are shown in Table I. The effective exchange field, coupling neighboring cells, is $H_{E}=A / M_{S} d^{2}$.

The equilibrium states are found using a self-consistent algorithm. ${ }^{9,10}$ For each value of the external field strength, the equilibrium configuration is found by seeking a set of directions of the moments in all cells $\left(\hat{m}_{i}, i=1, \ldots, N\right)$ which makes the torque in each one equal to zero, within reasonable numeric precision. Convergence is checked to guarantee a maximum torque of $10^{-26} \mathrm{~J}$ in any one of the cells. ${ }^{9}$

In Fig. 1 we show the hysteresis curves of interface biased and unbiased (in the inset) $170 \mathrm{~nm} \times 80 \mathrm{~nm} \times 30 \mathrm{~nm}$ iron elliptical nanoelements, for an interface field of $H_{\text {int }}=8.17 \mathrm{kOe}$. For the unbiased nano-element, the magnetization reversal is mediated by the nucleation of a vortex pair aligned along the major axis direction with the same chirality, separated by an antivortex, as shown in the panels (a) and (b) for the spin maps at the upper and lower branch reversal fields $H=-0.3 \mathrm{kOe}$ and $H=0.3 \mathrm{kOe}$, respectively. For a higher external field, the region aligned with the external field increase (marked with a white arrow) increases, and the vortex pair plus the antivortex structure is expelled at the vanishing domain side of the nano-element. Along this route, starting at the reversal field until annihilation, the vortex core distance is kept constant at around $50 \mathrm{~nm}$.

The interface field inhibits the antivortex formation and the reversal process involves the nucleation of a vortex pair with opposite chirality, separated by a magnetic domain. The vortex pair is not aligned along the major axis direction.
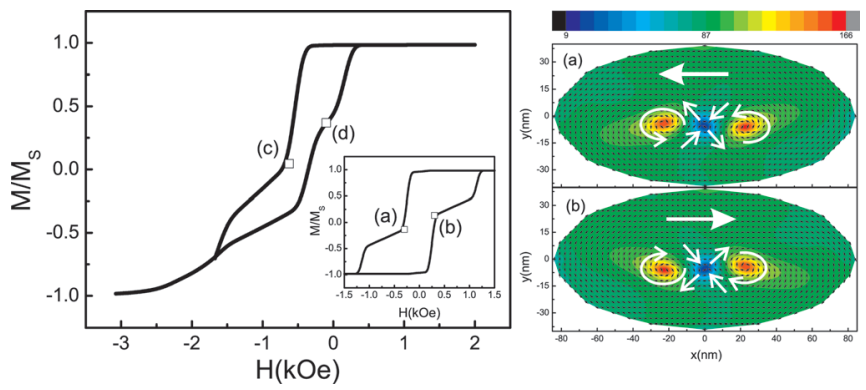

FIG. 1. (Color online) Hysteresis curves for an interface biased and unbiased (inset) $170 \mathrm{~nm} \times 80 \mathrm{~nm} \times 30 \mathrm{~nm}$ iron nano-element. The panels show the magnetic states at the vortex pair nucleation fields for the unbiased nano-element for (a) $H=-0.3 \mathrm{kOe}$ and (b) $H=0.3 \mathrm{kOe}$. The color bar code indicates the polar angle.
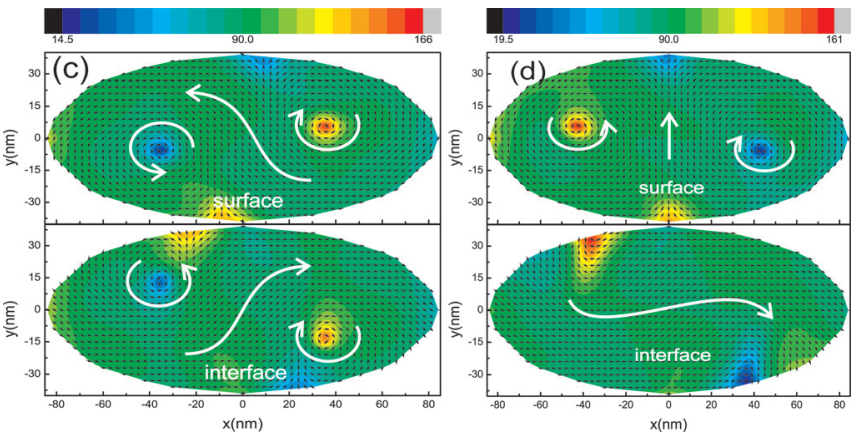

FIG. 2. (Color online) Magnetic states at vortex pair nucleation fields along the hysteresis loop of an interface biased $170 \mathrm{~nm} \times 80 \mathrm{~nm} \times 30 \mathrm{~nm}$ iron elliptical nano-element. The left and right panels show the spin maps at the points (c) for $H=-0.625 \mathrm{kOe}$ and (d) $H=-0.1 \mathrm{kOe}$, respectively. The color bar code indicates the polar angle.

Instead, at the reversal field $(H=-0.625 \mathrm{kOe})$, in the upper branch [marked (c) in Fig. 1] the central domain at the interface turns to the interface field direction (see bottom left panel in Fig. 2), whereas in the surface layer the central domain turns to the external field directions (see top left panel in Fig. 2).

By further increasing the external field in the direction opposite to the interface field the vortices are pulled in opposite directions and are eventually expelled at opposite sides of the nano-element. Along the way to saturation in the direction opposite to the interface field the distance between the vortex cores changes from about $74 \mathrm{~nm}$ at the nucleation field $(H=-0.625 \mathrm{kOe})$, to about $81 \mathrm{~nm}$ at the annihilation field $(H=-1.5 \mathrm{kOe})$.

The lower branch reversal [marked (d) in Fig. 1] occurs at $H=-0.1 \mathrm{kOe}$, with the interface layer almost aligned with the interface field direction (see bottom right panel in Fig. 2) and a vortex pair with opposite chirality separated by a diamond domain perpendicular to the external field (see top right panel in Fig. 2). By further increasing the external field, the central domain turns to the external field direction, pushing the vortices in opposite directions until they are expelled from the nano-element.

In Fig. 3 we show the hysteresis curves for interface biased and unbiased (in the inset) $200 \mathrm{~nm} \times 100 \mathrm{~nm} \times 20 \mathrm{~nm}$ Permalloy ${ }^{\mathrm{TM}}$ elliptical nano-element for an interface field of $H_{\text {int }}=6.25 \mathrm{kOe}$. As seen in the inset of Fig. 3, the magnetization reversal occurs via uniform rotation for the unbiased nano-element as shown by the square hysteresis loop.

The reversal of the interface biased nano-element involves nucleation of vortex pairs. As shown in Fig. 3 there is a drop in the magnetization to about almost zero at an external field value of $-1.5 \mathrm{kOe}$, and a subsequent reorientation, at $H=-0.3 \mathrm{kOe}$, to near saturation value in the reverse direction.

In the central panel we show the magnetic state for $H=-1.5 \mathrm{kOe}$ at the reversal field of the upper branch. Vortex pairs form at the surface and interface layer, and separated domains turned slightly toward the external field and interface field directions, respectively. The vortex pairs at the surface and interface are displaced, and the distance between both vortices is controlled by the strength of the external field. 

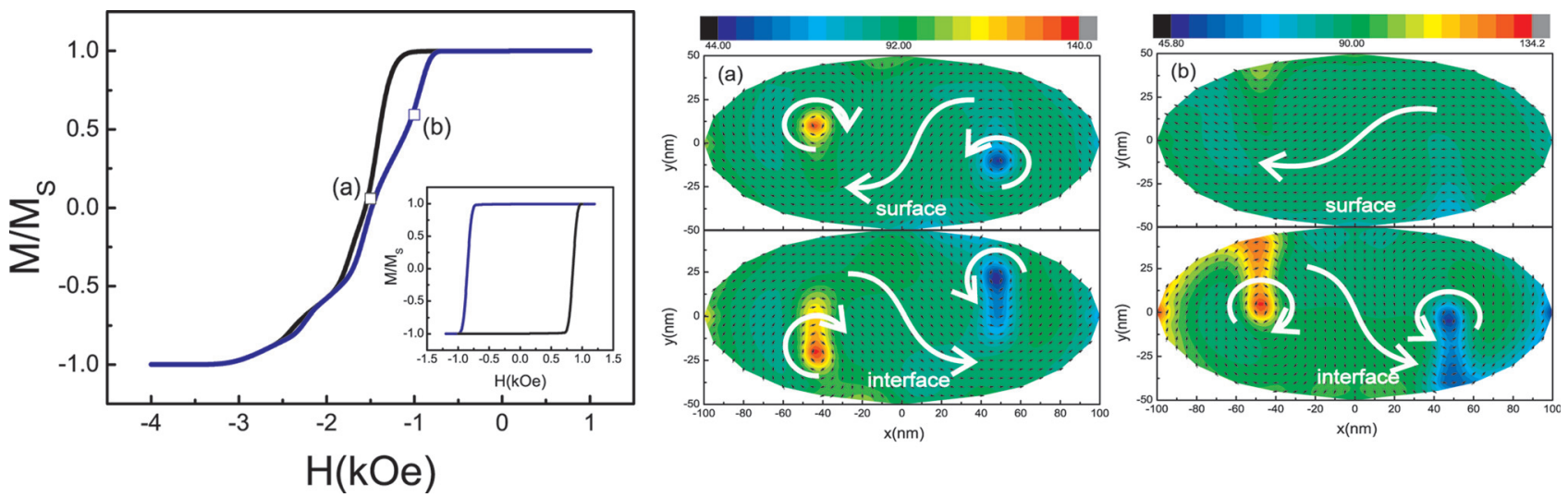

FIG. 3. (Color online) Hysteresis curves for interface biased and unbiased (inset) $200 \mathrm{~nm} \times 100 \mathrm{~nm} \times 20 \mathrm{~nm}$ Permalloy ${ }^{\mathrm{TM}}$ elliptical nano-element, for an interface field of $H_{\text {int }}=6.25 \mathrm{kOe}$. The central and right panels show the magnetic states at the vortex pair nucleation fields (a) $H=-1.5 \mathrm{kOe}$ and (b) $H=-1.0$ kOe. The color bar code indicates the polar angle.

In the right panel of Fig. 3 we show the magnetic state at the reversal in the lower branch, for $H=-1 \mathrm{kOe}$. It is intriguing that, contrary to intuition, the interface layer accommodates a pair of vortices of opposite chirality and polarity, whereas in the surface layer (top right panel), the magnetization is almost uniform. Howsaturation occurs along the external field direction involves the rotation of the surface domain as well as that of the central domain at the interface layer expelling the vortices at opposite sides of the nano-element.

In summary we have shown that interface biasing favors vortex pair nucleation along the hysteresis loop of iron and Permalloy ${ }^{\mathrm{TM}}$ elliptical nano-elements with geometrical dimensions in the few-100 $\mathrm{nm}$ range. Also, the distance as well as the relative chirality of vortex pairs in iron nano-elements may be affected by the interface exchange energy. We also notice that the vortex pairs form with opposite core polarity when there is a domain between them. However, as shown in Fig. 1 the vortex cores have the same polarity when separated by an antivortex of opposite polarity.

The key point is that vortex pair nucleation allows a reduction of the dipolar energy, as well as the Zeeman and interface exchange energy.

Vortex pair nucleation is favored by large values of the saturation magnetization, which demand a small surface charge at the lateral surfaces of the nano-element. Furthermore, it is also favored by large values of the interface field strength, which requires large fractions of the interface spins aligned with the interface field.
Our results suggest using the interface bias field as a possible manner to control the relative vortex chirality and the distance between the vortex cores. This might be a promising way to tailor synchronization of vortex pair dynamics for nano-oscillator applications.

The authors acknowledge support from CNPq, CAPES, and FAPERN. The work of A. S. Carrico and A. L. Dantas were supported by CNPq Grant Nos. 350773 and 309676.

${ }^{1}$ T. Shinjo, T. Okuno, R. Hassdorf, K. Shigeto, and T. Ono, Science 289, 930 (2000).

${ }^{2}$ A. Hubert and R. Schäfer, Magnetic Domains: The Analysis of Magnetic Microstructures (Springer, Berlin, 1998).

${ }^{3}$ J. Nogués and I. K. Schuller, J. Magn. Magn. Mater. 192, 203 (1999).

${ }^{4}$ J. Nogués, J. Sort, V. Langlais, V. Skumryev, S. Surinach, J. S. Munozb, and M. D. Baró, Phys. Rep. 422, 65 (2005).

${ }^{5}$ J. Sort, K. S. Buchanan, J. E. Pearson, A. Hoffmann, E. Menéndez, G. Salazar-Alvarez, M. D. Baró, M. Miron, B. Rodmacq, B. Dieny, and J. Nogués, J. App. Phys. 103, 07C109 (2008).

${ }^{6}$ A. Hoffmann, J. Sort, K. S. Buchanan, and J. Nogués, IEEE Trans Magn 44, 1968 (2008).

${ }^{7}$ O. Fruchart, J. P. Nozieres, W. Wernsdorfer, D. Givord, F. Rousseaux, and D. Decanini, Phys. Rev. Lett. 82, 1305 (1999).

${ }^{8}$ R. D. McMichael, J. Eicke, M. J. Donahue, and D. G. Porter, J. Appl. Phys. 87, 7060 (2000).

${ }^{9}$ G. O. G. Rebouças, A. S. W. T. Silva, A. L. Dantas, R. E. Camley, and A. S. Carriço, Phys. Rev.B 79, 104402 (2009).

${ }^{10}$ A. L. Dantas, G. O. G. Rebouças, A. S. W. T. Silva, and A. S. Carriço, J. Appl. Phys. 97, 10K105 (2005).

${ }^{11}$ M. Grimsditch et al., Phys. Rev. B 69, 174428 (2004).

${ }^{12}$ F. Porrati et al., Appl. Phys. Lett. 85, 3157 (2004).

${ }^{13}$ M. H. Park et al., Phys. Rev. B 73, 094424 (2004). 